\title{
An edge detector based on artificial neural network with application to hybrid Compact-WENO finite difference scheme
}

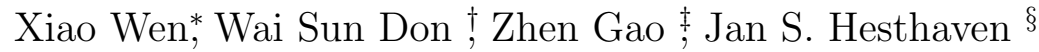

\begin{abstract}
A new approach is proposed to detect edges based on an artificial neural network (ANN). Some elementary continuous and discontinuous functions interpolated in the polynomial space and their continuity are used as the training sets to train a back propagation neural network containing two hidden layers. The ANN edge detector is used to detect the edges in an image and the locations of discontinuity in the hybrid fifth order Compact-WENO nonlinear (Hybrid)scheme for solving hyperbolic conservation laws with solutions containing both discontinuous and complex fine scale structures. Several classical examples in the image processing show that ANN edge detector can capture an edge accurately with fewer grid points than the classical multi-resolution (MR) analysis. Furthermore, as oppose to the M$\mathrm{R}$ analysis, ANN edge detector is robust with no problem dependent parameter, in addition to being accurate and efficient. The performance of the Hybrid scheme with the ANN edge detector is demonstrated with several one and two-dimensional benchmark examples in shallow water equations and Euler equations.
\end{abstract}

\section{Keywords}

Artificial neural network, Edge detection, Hybrid scheme, Hyperbolic conservation laws.

\section{Introduction}

Characteristic-wise weighted essentially non-oscillatory (WENO) conservative finite difference schemes, as a class of high order/resolution nonlinear scheme for the solutions of hyperbolic conservation laws in the presence of shocks and small scale structures, was initially developed by [11] (for details and history of the WENO scheme, see [2, 3, 22] and references therein). However, the WENO scheme is fairly complex to implement, computationally expensive and too dissipative for certain classes of problems. A natural way to alleviate some of these difficulties is to construct a hybrid scheme conjugating a nonlinear WENO scheme in the non-smooth stencils with a linear (Compact, fintie difference, or spectral) scheme in the smooth stencils $[4,8,14,16,20]$. The key issue in any hybrid scheme is the design of an accurate, robust,

\footnotetext{
${ }^{*}$ Key Laboratory of Physical Oceanography, Ocean University of China, Qingdao, China. E-Mail: xiaowen_ouc@63.com

${ }^{\dagger}$ School of Mathematical Sciences, Ocean University of China, Qingdao, China. E-Mail: donwaisun@outlook.com

${ }^{\ddagger}$ corresponding author: School of Mathematical Sciences, Ocean University of China, Qingdao, China. E-Mail: zhengao@ouc.edu.cn

${ }^{\S}$ École Polytechnique Fédérale de Lausanne (EPFL), Route Cantonale, 1015 Lausanne Switzerland. E-Mail: jan.hesthaven@epfl.cn
} 
and efficient high order shock detection algorithm that is capable of determining the smoothness of the solution at any given grid point and at any given time.

In the last two decades, many shock detection methods for the hybrid scheme for solving hyperbolic conservation laws have been developed. Costa et al. [4,5] described the arbitrary order multi resolution (MR) analysis by Harten [10] for identifying the non-smooth and smooth stencils in the hybrid scheme. Generally speaking, high order MR analysis is preferable to a low order shock detector and the conjugate Fourier shock detector in the high order hybrid scheme for its efficiency (with its $O(N)$ floating point operations count). Don et al. [7] proposed the conjugate Fourier shock detector which uses the conjugate Fourier partial sum and its derivative in detecting discontinuities in a function. It is a parameter free technique and successfully has been employed in the high order hybrid Compact-WENO finite difference scheme (Hybrid) for solving hyperbolic conservation laws. MR analysis perform well as long as a suitable, problem-dependent parameter is chosen. This parameter is used in a threshold which decides whether or not to label an element as a troubled cell. Gao et al.[9] combined the Tukeys boxplot method and MR analysis for solving the hyperbolic conservation laws with the hybrid scheme. The improved Tukeys boxplot method essentially removes the need of specifying the MR Tolerance $\epsilon_{M R}$, and thus greatly improves the robustness of the hybrid scheme.

Artificial Neural Networks (ANN) is an interconnection network composed of simple neurons. The neurons that make up such a parallel and extensive network are often adaptable. The organization of the network can "simulate the interaction of biological nervous system with real world objects" [12]. Back Propagation (BP) network is one of the most widely used neural network. It was proposed by Rumelhart and McCelland [19] in 1986. It is a multi-layer feedforward network trained by the error back propagation algorithm. BP neural network can learn and store a large number of related mathematical models without revealing and describing the mathematical equation of the mapping relationship between input and output beforehand.

Ray and Hesthaven [17] proposed a new approach to detect discontinuities using ANN to find a troubled-cell where the limiter should be used in the Runge-Kutta discontinuous Galerkin (RKDG) method for solving hyperbolic conservation laws. The training process is performed offline on a robust data set consisting of canonical samples characterizing the local solution structures of conservation laws. The numerical results demonstrate that the ANN troubled-cell indicator does not need the parameters related to the problem and performs better than the TVB limiter with an optimally chosen parameter $M$.

The main goal in this study is to construct an ANN edge detector, which can be used in the image processing and in the Hybrid scheme for the solutions of hyperbolic conservation laws. We first improve the training sets in [17] with an extensive set of basis function in the polynomial space $\mathbb{P}^{k}$ (here, $k=5$ is the optimal order of the WENO finite difference scheme, which is used in the Hybrid scheme). Secondly, three mean values in the RKDG method is replaced by five point values of function in data sets in the Hybrid scheme. The detailed training process including the input, output, network structure and data set will be shown in Section 2. Last but not least, we construct a two hidden layers BP network with $\{8,4\}$ neurons, which uses less training time than that in [17] containing five hidden layers with $\{256,128,64,32,16\}$ neurons. The accuracy of the ANN edge detector is demonstrated by capturing an edge in the classical images with about two grid points. Finally, we investigate the applicability of the ANN edge detector in high order Hybrid scheme for solving hyperbolic conservation laws. Compared with the Hybrid scheme with the classical MR analysis [4, 27] (Hybrid-MR) in terms of accuracy and efficiency, the Hybrid scheme with the ANN edge detector (Hybrid-ANN) use similar CPU time, but usually generate much sharper discontinuous stencils. Its percentage of the WENO reconstruction is only half of that in the Hybrid-MR scheme. 
This paper is organized as follows. In Section 2, we briefly introduce the BP network and present the algorithm of the ANN edge detector. In Section 3, representative examples in the edge detection of the one-dimensional function with multiple discontinuities and two-dimensional Shepp-Logan image are given. In Section 4, the Hybrid scheme is briefly reviewed. In Section 5, several one- and two-dimensional shocked problems are simulated to illustrate the performance of the Hybrid scheme. Concluding remarks are given in Section 6. The sixth order compact finite difference scheme, fifth WENO-Z finite difference scheme and MR analysis are given in Appendix.

\section{Artificial Neural Network edge detector}

In this section, we will design an edge detection algorithm based on ANN. We focus on a Back Propagation (BP) neural network [19], which is one of the most widely used neural network at present. BP network can learn and store a large number of related mathematical models without revealing and describing the mathematical equation of the mapping relationship between input and output beforehand. Its network model topology includes input layer, hidden layers and output layer, which is shown in Fig. 1.

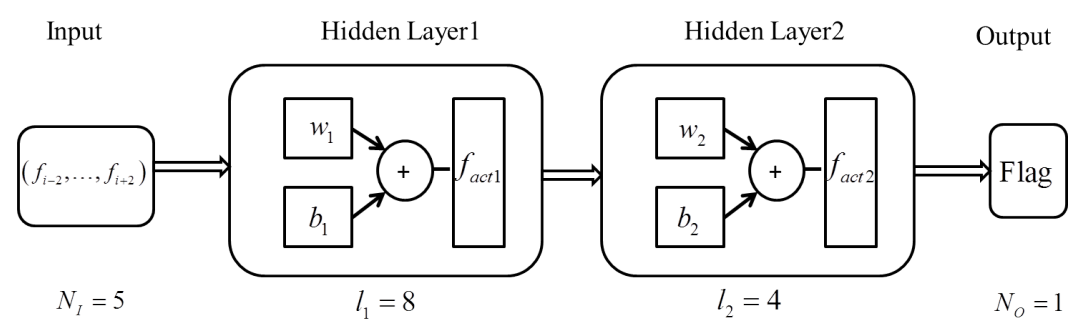

Figure 1: BP neural network for edge detection.

The learning process of BP neural network can be divided into two steps. They are the forward calculation process and the error back propagation process. In the forward calculation process, the preprocessed input is entered into the network from the input layer, and then calculated layer by layer through the hidden layer. Finally, it is transferred to the output layer. The state of each layer of neurons only affects the state of the next layer of neurons. If the output value of the output layer does not satisfy the expectation, it enters the error back propagation process. The error signal returns along the original connection path, and adjusts the weights and thresholds of each layer of the network one by one until the input layer is reached, and then repeats the forward calculation. These two processes alternate and repeat, constantly adjusting and updating the weights and thresholds of each layer. When the network error is minimal or has met the expectations, the learning process ends.

\section{$2.1 \quad$ Input and Output}

In this work, the number of neurons in the input layer is $N_{I}=5$, and the input value of the network is selected as the function values $\mathbf{f}=\left(f_{i-2}, f_{i-1}, f_{i}, f_{i+1}, f_{i+2}\right)$ at the five uniformly spaced points $\left(x_{i-2}, x_{i-1}, x_{i}\right.$, $\left.x_{i+1}, x_{i+2}\right)$ with a constant cell size $\Delta x$ around $x_{i}$. A linear normalization algorithm is used to preprocess 
the input data and to normalize the input function values into $[-1,1]$ :

$$
\hat{f}_{j}=\frac{f_{j}}{\max _{k=-2, \ldots, 2}\left(\left|f_{i+k}\right|, 1\right)}, \quad j=i-2, \ldots, i+2,
$$

where $\hat{\mathbf{f}}$ is a normalized vector of $\mathbf{f}$.

The output value $O_{i}$ represents the smoothness of $f_{i}$, and the number of neurons in the output layer is $N_{O}=1$. Rounding $O_{i},\left[O_{i}\right]$ is used to mark whether the function is discontinuous or smooth at the point of interest $x_{i}$ by a Flag $_{i}$ as

$$
\operatorname{Flag}\left(x_{i}\right)=\text { Flag }_{i}=\left[O_{i}\right]=\left\{\begin{array}{cc}
1, & \text { discontinuous } \\
0, & \text { smooth }
\end{array}\right.
$$

\subsection{Network Structure}

As an operation model, neural network is composed of a large number of nodes (or neurons) connected with each other. The number of neurons and the selection of activation function play an important role in the neural network. The BP neural network constructed for the edge detection in this study contains two hidden layers $H_{1}$ and $H_{2}$, and the number of neurons of hidden layer is set to $\left\{N_{H_{1}}, N_{H_{2}}\right\}=\{8,4\}$.

The purpose of activation function is to introduce nonlinearity into the network model. We choose the Relu activation function $f(x)=\max (0, x)$ in the hidden layers $H_{1}$ and $H_{2}$, which will expand the feature effect in the process of cycling. In the output layer, the sigmoid function $\left(f(x)=\frac{1}{1+e^{-x}}\right)$ is used to map the output number $O_{i}$ to the range $(0,1)$. Thus, $O_{i}$ means the probability that the point $x_{i}$ is identified as a discontinuous point or not.

\subsection{Data Set}

As mentioned in Section 2.1, the input values $\mathbf{f}=\left(f_{i-2}, f_{i-1}, f_{i}, f_{i+1}, f_{i+2}\right)$ of the data set are the point values of the function $f(x)$ at a point $x_{i}$ and $\left(x_{i-2}, x_{i-1}, x_{i}, x_{i+1}, x_{i+2}\right)$ are the locations of the grid point (assuming to be uniformly spaced with cell size $\Delta x$ in this study) in the function definition domain $D$. By reasonably selecting functions and parameters, the function values in the data set are normalized in $[-1,1]$. There are four steps when creating a data set:

1. Choose a function $f(x)$ and its parameters in Table I.

2. Pick a point $x_{i}$ and set a meshsize $\Delta x$ such that $\left(x_{i}-2 \Delta x, x_{i}+2 \Delta x\right) \in D$.

3. Compute the function values $\mathbf{f}=\left(f_{i-2}, f_{i-1}, f_{i}, f_{i+1}, f_{i+2}\right)$.

4. Set the true output Flag $_{i}=O_{i}$ where $O_{i}=1$ if the function is discontinuous at the point $x_{i}$ and $O_{i}=0$ otherwise.

The number of functions, parameter values, definition fields, discontinuity marks and the number of functions selected in the data set are shown in Table I. $75 \%$ of the data set is taken as the training set and the 
Table I: Data set: Functions $f(x)$, parameter values, definition domains $D$, discontinuous identifiers Flag and the number of functions in the data set.

\begin{tabular}{crrrr}
\hline$f(x)$ & \multicolumn{1}{r}{ parameter } & \multicolumn{1}{c}{$D$} & Flag & number \\
\hline $\sin (2 \pi x)$ & $k \in(-10,10)$ & $\left(-\frac{1}{k}, \frac{1}{k}+4 \Delta x\right)$ & 0 & 0 \\
$k x$ & $k \in(-10,10)$ & $\left(-\frac{1}{k}, \frac{1}{k}+4 \Delta x\right)$ & 1 & 100000 \\
$k|x|$ & $k \in(-10,10), a=2, \ldots, 5$ & $\left(-\frac{1}{k}, \frac{1}{k}+4 \Delta x\right)$ & 0 & 40000 \\
$k x^{a}$ & $l \in(-1,1), r \in(-1,1)$ & $(0,1)$ & 1 & 80000 \\
$c$ & $c \in(-1,1)$ & $(0,1)$ & 0 & 20000 \\
$l\left(x<x_{0}\right)+r(x>x 0)$ & & & &
\end{tabular}

rest $25 \%$ as the test set. Once we obtain the trained network, the ANN edge detector can be designed by the algorithm as shown as follow.

Algorithm: (ANN edge detector)

1. Create the data sets $\mathbf{f}$ and $O$ in Section 2.3 .

2. Training the BP network constructed in Section 2.2, get and save the corresponding relationship between input and output, that is the network $\mathcal{N}$.

3. For the function value $\tilde{f}_{i}$ at the point $\tilde{x}_{i}$ need to be judged smooth or not, compute and normalize $\tilde{\mathbf{f}}=\left(\tilde{f}_{i-2}, \tilde{f}_{i-1}, \tilde{f}_{i}, \tilde{f}_{i+1}, \tilde{f}_{i+2}\right)$ to obtain $\widehat{\mathbf{f}}$ to be used as the network input. The output $\tilde{O}$ and $\operatorname{Flag}_{i}=\left[\tilde{O}_{i}\right]$ are computed through the saved network $\mathcal{N}$.

Table II shows the accuracy for identifying the discontinuities of the last $25 \%$ of test data set through the saved network $\mathcal{N}$ after $k$ trainings. It can be observed that the accuracy of each training session is above $96 \%$.

Table II: 10 times accuracy test for $25 \%$ test set.

\begin{tabular}{c|cccccccccc}
\hline$k$ & 1 & 2 & 3 & 4 & 5 & 6 & 7 & 8 & 9 & 10 \\
\hline Accuracy & $97.8 \%$ & $97.3 \%$ & $98.3 \%$ & $96.8 \%$ & $97.4 \%$ & $97.2 \%$ & $97.3 \%$ & $96.7 \%$ & $97.1 \%$ & $97.4 \%$ \\
\hline
\end{tabular}

\section{Numerical Results for Edge Detection}

In this section, we will present some one-dimensional and two-dimensional benchmark images to illustrate the ability of discontinuity capture in image edge detection. The edge set $S$ here contains all the centers which are identified as the edges/boundaries/sharp gradients, which means $S=\left\{x_{i} \mid\right.$ Flag $\left.\left(x_{i}\right)=1, \quad \forall i\right\}$. 


\subsection{One-dimensional Multi-Wave Functions}

Multi-wave function includes smooth Gauss function, discontinuous square function, piecewise linear triangular function and continuous elliptic function. This function tests the abilities of any discontinuity detector in identifying a smooth function and discontinuities in the function and/or its first derivative under various grid resolutions. Its function expression is as follows:

$$
f(x)= \begin{cases}\frac{1}{6}[G(x, \beta, z-\delta)+4 G(x, \beta, z)+G(x, \beta, z+\delta)], & x \in[-0.8,-0.6] \\ 1, & x \in[-0.4,-0.2] \\ 1-|10(x-0.1)|, & x \in[0,0.2] \\ \frac{1}{6}[F(x, \alpha, a-\delta)+4 F(x, \alpha, a)+F(x, \alpha, a+\delta)], & x \in[0.4,0.6] \\ 0, & \text { else },\end{cases}
$$

where $G(x, \beta, z)=e^{-\beta(x-z)^{2}}, F(x, \alpha, a)=\sqrt{\max \left(1-\alpha^{2}(x-a)^{2}, 0\right)}$, and $z=-0.7, \delta=0.005, \beta=\frac{\log 2}{36 \delta^{2}}$, $a=0.5, \alpha=10$.

Fig. 2 shows the detection results, where the red dots and blue triangles denote the set of points in the edge set $S$ detected by the ANN edge detector and the MR analysis with $\epsilon_{M R}=2 \times 10^{-2}$. The locations of all the discontinuities (both in the jump in the function and its first derivative) of the multi-wave function Eq. (3) are identified more clearly and accurately by the ANN edge detector. For the step function, the location of jump in the function at $x=-0.4$ and $x=-0.2$ are accurately determined by the ANN edge detector, due to the step function $l\left(x<x_{0}\right)+r(x>x 0)$ in training set. Because of the absolute value function $k|x|$, the ANN edge detector also can capture jump in the first derivative at $x=-0.7$ and $x=0.1$. No false positive result from the ANN edge detector is observed in both the lower $(N=150)$ and higher $(N=250)$ resolution solutions.
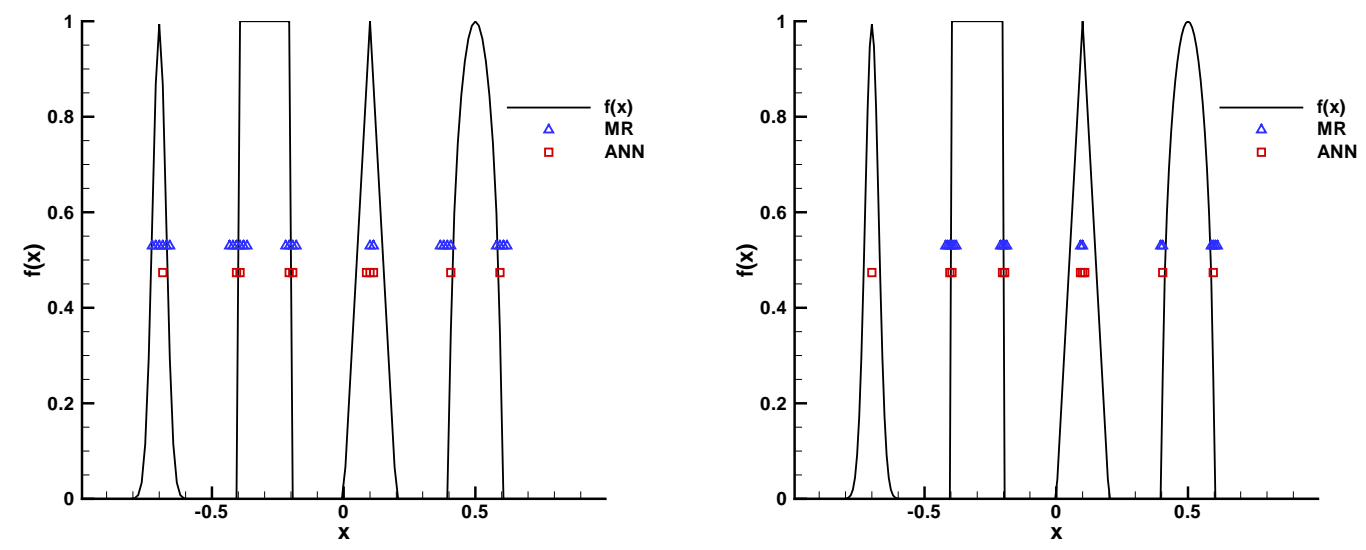

Figure 2: Edge detection of multi-wave function by the ANN edge detector and the MR analysis with (Left) $N=150$ and (Right) $N=250$. 


\subsection{One-dimensional Piecewise Function}

In this example, a piecewise function with five discontinuities of different jump heights is considered. As shown in Fig. 3, the degree of edge (strength) of the function varies greatly in different regions. The jumps of the function are ranged from 1 to 16. This example tests the ability of the edge detector in capturing edges of a multi-scales function. The red dots in Figure 3 show the detection results of ANN edge detector and the blue triangles show the results of MR analysis with $\epsilon_{M R}=1 \times 10^{-1}$. The results show that present method can not only capture every discontinuity, but also only with two grid points around it. The MR analysis, however, needs at least four grid points. Hence, ANN edge detector is more accurate than the MR analysis.

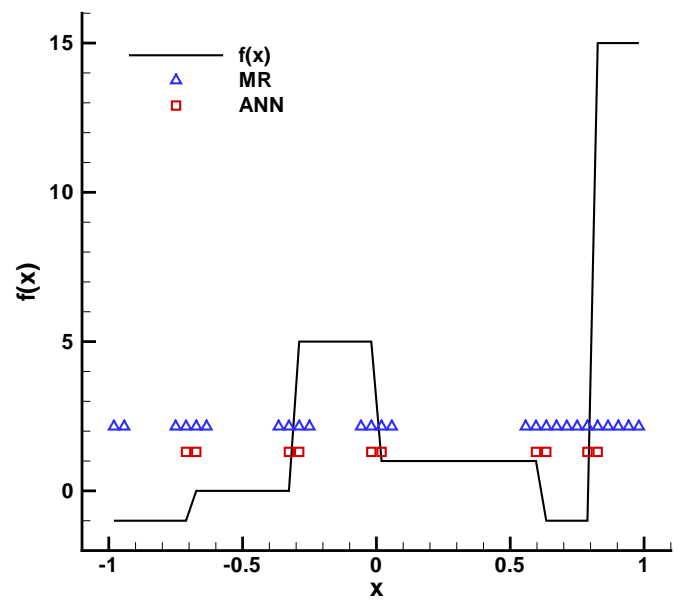

Figure 3: Discontinuity detection of a piecewise function by the ANN edge detector and the MR analysis with $N=50$.

\subsection{Edge Detection of Two-Dimensional Image}

Next, we test the performance of the ANN edge detector in the two-dimensional image edge detection. In the calculation process, the dimension splitting method is used. That is, edges are detected along the $x-$ and $y$ - directions respectively, and the final edge set is the union of the edge sets detected along the $x-$ and $y$ - directions.

Shepp-Logan image is a classic image, which is used to simulate the gray image of human brain, and it is also a two-dimensional piecewise function. Shepp-Logan image consists of a large ellipse (representing the brain, the corresponding function value is large) and several small ellipses (representing some characteristic structures of the brain, the corresponding function value is small). The intensity of each edge of the image varies greatly, where the minimum and maximum jump are 0.1 and 1 respectively, so it is often used to test the ability of edge detection method in capturing edges with muti-scales discontinuous. Furthermore, the image is densely distributed, and can also be used to test the ability of the edge detection method to capture "continuous" discontinuities. As shown in Fig. 4, under the image resolution $N \times M=256 \times 256$, the ANN edge detector can accurately capture both the large and small discontinuities simultaneously. 

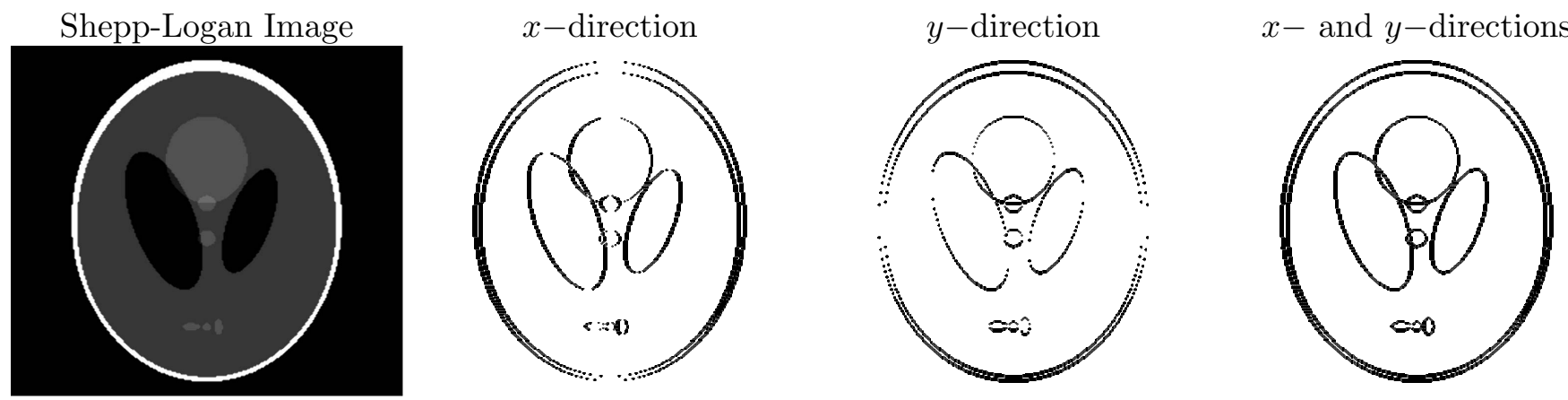

Figure 4: Edge detection of Shepp-Logan image with $N \times M=256 \times 256$. From left to right: the original image, the edges in $x$-direction, the edges in $y$-direction and the edges in both $x$ - and $y$-directions.

Fig. 5 shows the edge detection results of Shepp-Logan image along the $x$ - direction at $y=111$ using the ANN edge detector and the MR analysis with $\epsilon_{M R}=5.0 \times 10^{-5}$. The accuracy of edge detection can be observed more intuitively. Edge detection method based on artificial neural network not only can detect the edges with jump size of discontinuity accurately, but also perform well in detecting multiple "continuous" edges. The results show that the ANN edge detector behaves better than the MR analysis in identifying the locations of edges with a fewer grid points.

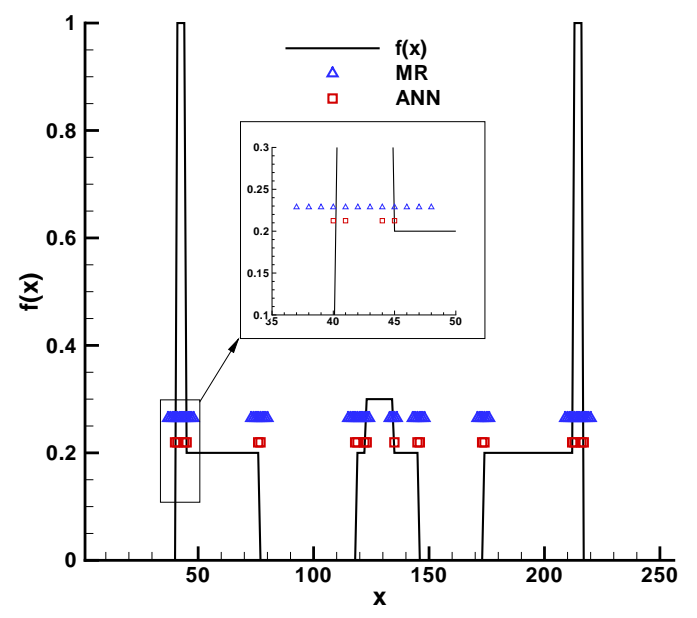

Figure 5: Detection results of Shepp-Logan image with $N \times M=256 \times 256$ along the $x$-direction at $y=111$.

The final detected edges of a sunflower and three other classical photos are shown in Fig. 6 respectively. One can easily see that the ANN edge detector works very well in detecting edges with both large and small jumps even when some photos contain very fine scale structures. 

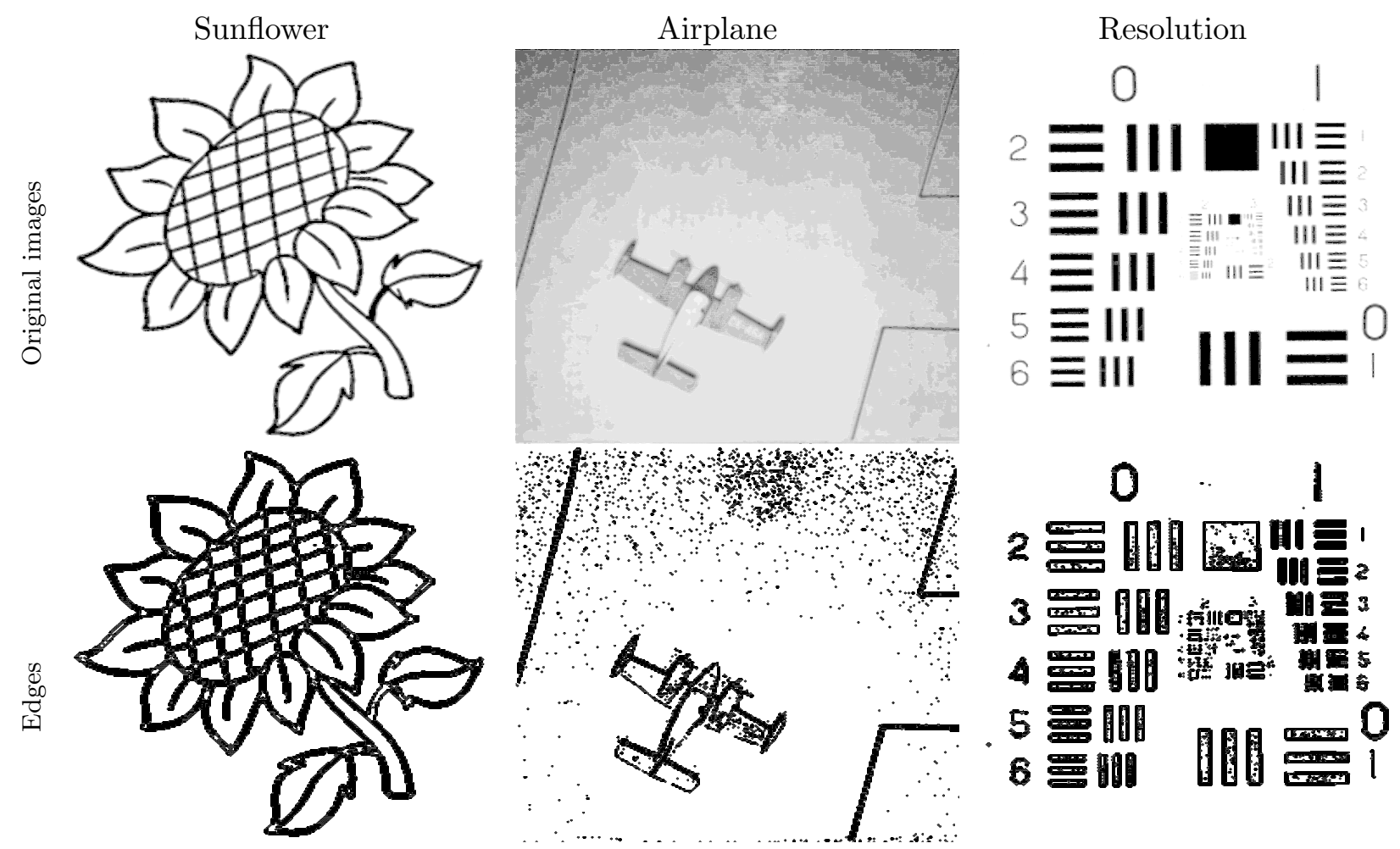

Figure 6: Final edges of images with $N \times M=256 \times 256$. (Top) The original images and (Bottom) the detected edges by the ANN edge detector. 


\section{Hybrid Scheme}

In this section, we present the temporal-spatial adaptive algorithm of the higher order hybrid CompactWENO finite difference scheme that is used for solving both the shallow water equations and the Euler equations $[9,7,27]$. The details for implementation of the numerical schemes (the sixth order compact finite difference scheme, the fifth order characteristic-wise WENO-Z finite difference scheme with the global Lax-Friedrichs flux splitting via the Roe eigensystem, the eighth order finite difference filtering and the third order Runge-Kutta TVD scheme) used in this study can be found in [7, 9, 27] and will be briefly introduced in Appendix. Other temporal-spatial adaptive hybrid algorithms using different shock-detection algorithms/troubled-cell indicators, for example $[4,8,9,14,16,20]$ can be used as an alternative method of $[7,9,27]$. Interested readers are referred to references and contained therein for the details.

The hybrid algorithm consists of four main steps that, 1) at the beginning of the Runge-Kutta time step, determine the smoothness of solution at a given grid point by a shock detection method, 2) markup the grid points with a Flag, 3) setup a buffer zone (blue circle in Fig. 7) around the non-smooth grid point, 4) compute the derivative of the flux with the WENO-Z scheme at the non-smooth stencils and with the central compact scheme otherwise, and 5) at the end of the Runge-Kutta time step, stabilize the solution by a finite difference filtering [23] in the smooth stencils if needed.

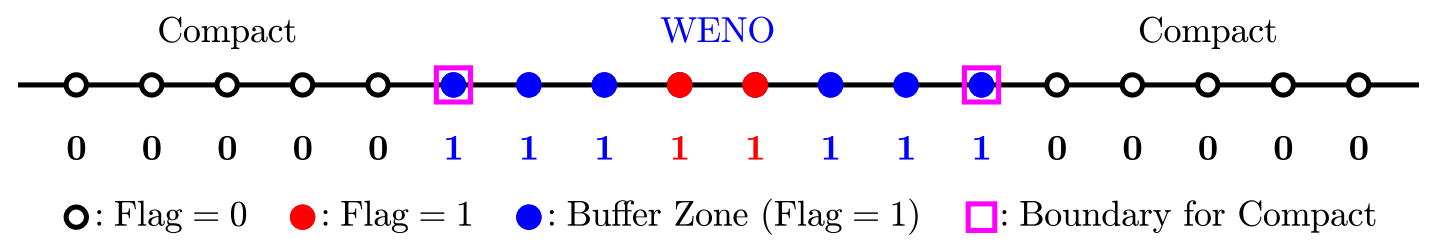

Figure 7: Diagram of the buffer zone.

In the following discussion, we shall denote the hybrid Compact-WENO finite difference scheme using the MR analysis and ANN edge detector as the Hybrid-MR scheme and Hybrid-ANN scheme respectively, and collectively refer to both schemes as the Hybrid schemes.

\section{Numerical Results for Hybrid scheme}

To demonstrate the performance of the Hybrid-ANN scheme, we present the numerical results of several shocked flow problems in the one- and two-dimensional shallow water equations and Euler equations. We shall provide the numerical solutions computed by the WENO-Z, Hybrid-MR and Hybrid-ANN schemes and give their corresponding CPU timing results. The WENO percentage is defined as the percentage of non-smooth stencils solved by the WENO-Z scheme in the whole domain. In all the examples below, the third order TVD Runge-Kutta method [2] with CFL $=0.45$ is used for the temporal evolution of the resulted ODE at each grid points. 


\subsection{Shallow water equation}

The two-dimensional shallow water equations take the form

$$
\mathbf{Q}_{t}+\mathbf{F}_{x}+\mathbf{G}_{y}=\mathbf{S}
$$

where the conservative variables $\mathbf{Q}$, the fluxes $\mathbf{F}$ and $\mathbf{G}$ in the $x$ - and $y$-directions, respectively, are

$\mathbf{Q}=(h, h u, h v)^{T}, \quad \mathbf{F}=\left(h u, h u^{2}+\frac{1}{2} g h^{2}, h u v\right)^{T}, \quad \mathbf{G}=\left(h u, h u v, h v^{2}+\frac{1}{2} g h^{2}\right)^{T}, \quad \mathbf{S}=\left(0,-g h b_{x},-g h b_{x}\right)^{T}$,

and $h$ is the water height, $(u, v)^{T}$ is the velocity vector, $b(x, y)$ is the bottom topography and $g=9.812$ is the gravitational constant. The well-balanced technique [26] is used in the Hybrid schemes to reformulate the source terms to maintain the exact C-property.

\subsubsection{One-dimensional Dam-breaking Problem over a Rectangular Bump}

In this section, a classical test case in [24] is selected to study the ability of hybrid schemes to capture shocks in complex situations. We simulate the one-dimensional dam-breaking problem on rectangular bulge, which involves unstable flow over discontinuous bottom topography

$$
b(x)= \begin{cases}8, & \text { if }|x-750| \leq 1500 / 8 \\ 0, & \text { otherwise }\end{cases}
$$

The initial conditions are

$$
h(x, 0)=\left\{\begin{array}{ll}
20-b(x), & \text { if } x \leq 750, \\
15-b(x), & \text { otherwise }
\end{array} \quad u(x, 0)=0 .\right.
$$

The final time is $t=15$. In Fig. 8, we compare the solutions computed by the Hybrid-ANN scheme and the Hybrid-MR scheme with $N=500$ cells. The temporal history of the WENO-Z reconstruction at each grid point computed by two Hybrid scheme are shown to demonstrate the performance of the Hybrid schemes in Fig. 9. In this example, although the water depth $h$ contains discontinuities at $x=562.5$ and $x=937.5$, it is clear that the essentially non-oscillatory solutions are obtained accurately and agree very well with the reference solution. As shown in Table III, the Hybrid schemes are faster, and at high resolution, can be almost three times faster than the WENO-Z scheme. Furthermore, the WENO percentage in the Hybrid-ANN scheme is only half of that in the Hybrid-MR scheme. It will be confirmed in the following examples.

\subsubsection{Two-dimensional Dam-breaking Problem}

Here, we investigate the performance of the Hybrid-ANN method in simulating the two-dimensional dambreaking problem. The computational domain is $[0,200]^{2}$. The width of the dam is set to be 5 and the breach is located from $x=97$ to $x=102$ and between $y=65$ and $y=135$. The boundary conditions are reflective at the top and bottom boundaries, and the inflow and outflow boundary conditions are imposed in the left and right boundaries respectively. 

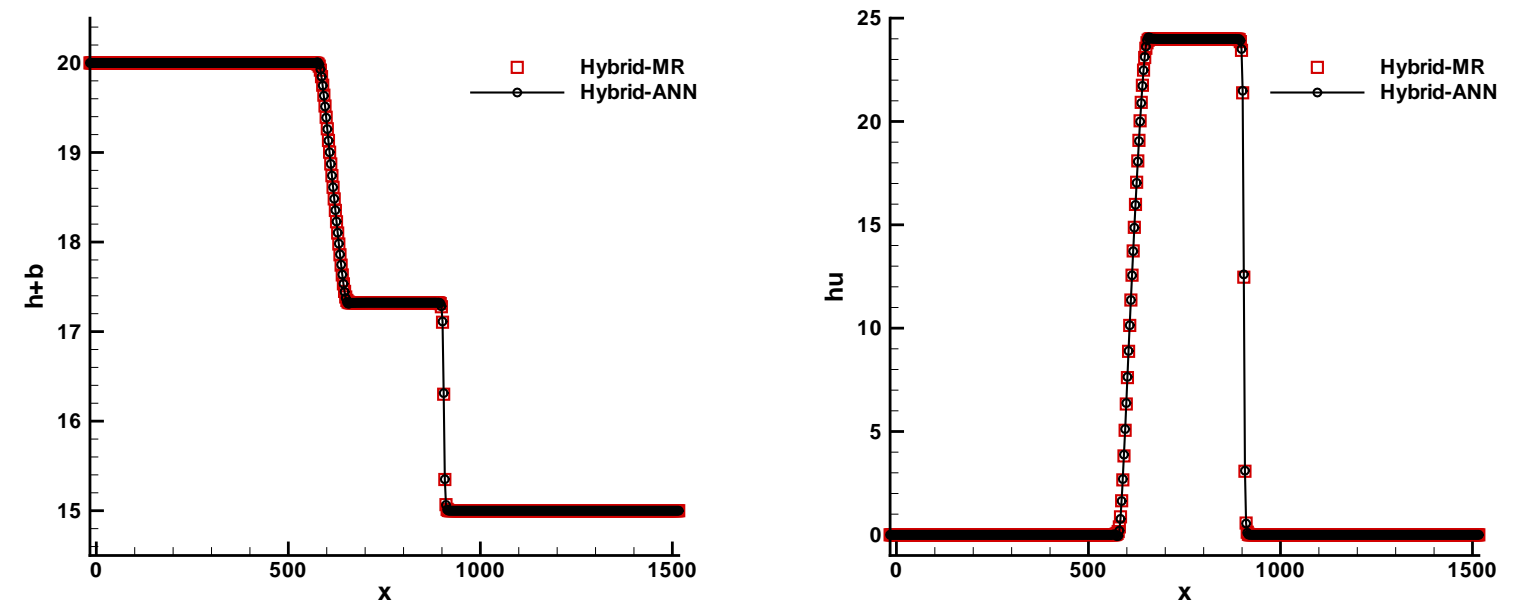

Figure 8: One-dimensional dam-breaking problem over a rectangular bump with $N=500$ cells. (Left) The water surface level $h+b$ and (Right) momentum $h u$ at time $t=15$.

Hybrid-MR

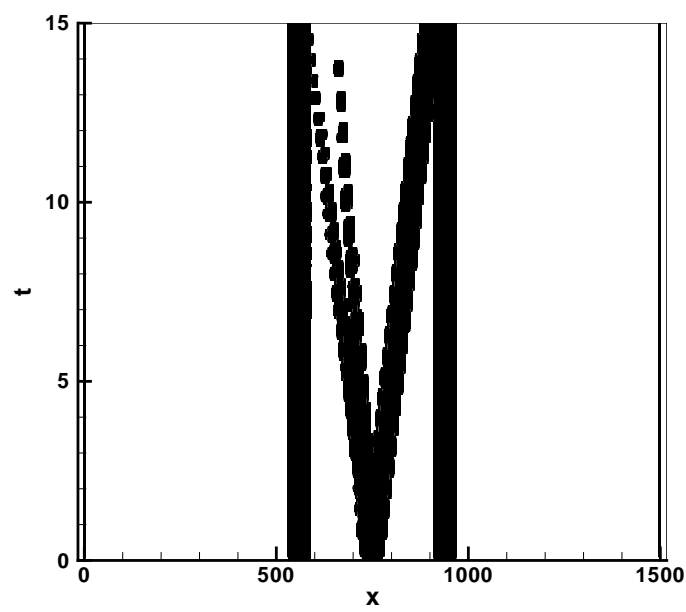

Hybrid-ANN

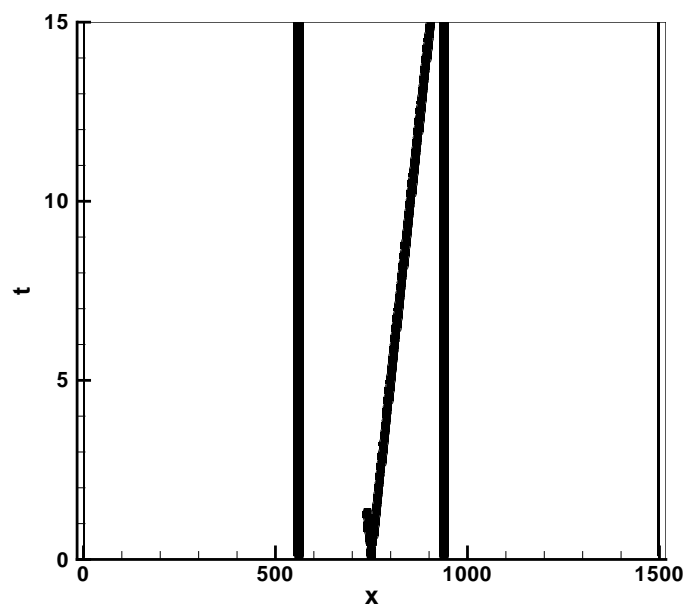

Figure 9: Temporal history of the WENO-Z reconstruction as computed by the Hybrid-MR scheme and Hybrid-ANN scheme. 
Table III: One-dimensional dam-breaking problem over a rectangular bump. The CPU timings, speedup factors (SF) and WENO percentage of the WENO-Z and Hybrid schemes.

\begin{tabular}{|c|c|c|c|c|c|c|c|}
\hline \multirow{2}{*}{$N$} & WENO & \multicolumn{3}{|c|}{ Hybrid-MR } & \multicolumn{3}{|c|}{ Hybrid-ANN } \\
\hline & seconds & seconds & $\mathrm{SF}$ & percentage & seconds & $\mathrm{SF}$ & percentage \\
\hline 100 & $5.6 \mathrm{E}-3$ & $4.1 \mathrm{E}-3$ & 1.36 & $45.5 \%$ & $2.6 \mathrm{E}-3$ & 2.15 & $20.8 \%$ \\
\hline 500 & $8.0 \mathrm{E}-2$ & $3.3 \mathrm{E}-2$ & 2.43 & $10.8 \%$ & $3.1 \mathrm{E}-2$ & 2.62 & $5.0 \%$ \\
\hline 5000 & 7.74 & 2.8 & 2.76 & $1.28 \%$ & 2.82 & 2.74 & $0.5 \%$ \\
\hline
\end{tabular}

The initial conditions are

$$
\begin{aligned}
& h(x, y, 0)=\left\{\begin{array}{ll}
10, & \text { if } x \leq 100 \\
5, & \text { otherwise }
\end{array},\right. \\
& h u(x, y, 0)=h v(x, y, 0)=0 .
\end{aligned}
$$

The water surface level and its velocity field are shown in Fig. 10 at time $t=7.2 \mathrm{~s}$. The sharp water front formed by the breaking of the dam is captured essentially oscillations free. The shear vortical structures generated at the tips of the breach is in a good agreement with those shown in [1].

Table IV shows a comparison on the CPU times and speedup factors of the WENO-Z scheme, Hybrid-ANN scheme and Hybrid-MR scheme with $\epsilon_{M R}=5 \times 10^{-4}$. We can clearly find that the Hybrid schemes allow a speedup up to a factor of two compared to the WENO-Z scheme. The WENO percentage of the ANN edge detector is smaller than the MR analysis.

$$
h+b
$$

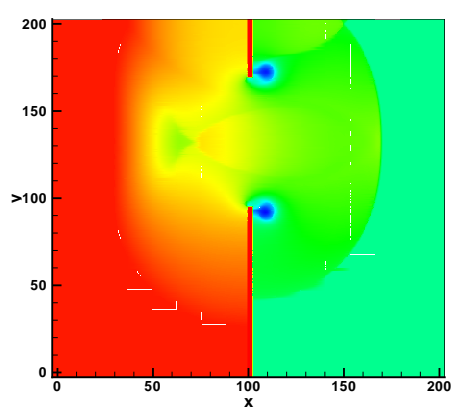

$\operatorname{Flag}_{x}$

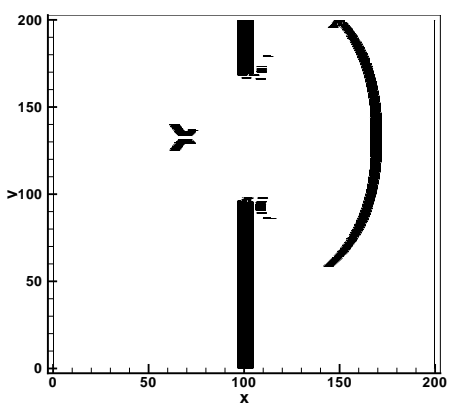

$\operatorname{Flag}_{y}$

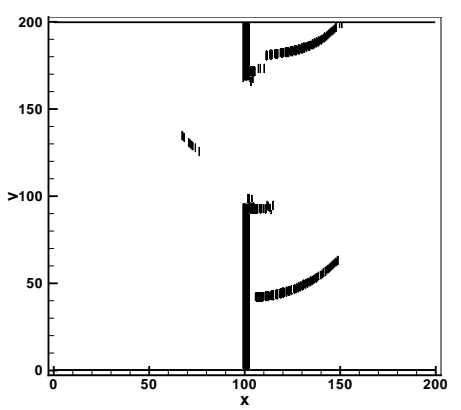

Figure 10: Two-dimensional dam-breaking problem. (Left) The water surface level $h+b$ and (middle) Flag $_{x}$ and (right) Flag $y$ identified by the ANN edge detector under resolution $400 \times 400$ at time $t=7.2$.

\section{$5.2 \quad$ Euler Equation}

We consider the two-dimensional Euler equations for gas dynamics in a conservation form:

$$
\mathbf{Q}_{t}+\mathbf{F}_{x}+\mathbf{G}_{y}=0
$$


Table IV: Two-dimensional dam-breaking problem. The CPU timings, speedup factors (SF) and WENO percentage of the WENO-Z and Hybrid schemes.

\begin{tabular}{|c|c|c|c|c|c|c|c|}
\hline \multirow{2}{*}{$N \times M$} & \multirow{2}{*}{$\begin{array}{l}\text { WENO } \\
\text { seconds }\end{array}$} & \multicolumn{3}{|c|}{ Hybrid-MR } & \multicolumn{3}{|c|}{ Hybrid-ANN } \\
\hline & & seconds & $\mathrm{SF}$ & percentage & seconds & $\mathrm{SF}$ & percentage \\
\hline $200 \times 200$ & 108.9 & 48.7 & 2.24 & $25.3 \%$ & 44.9 & 2.43 & $12.2 \%$ \\
\hline $400 \times 400$ & 428.5 & 144.6 & 2.96 & $14.5 \%$ & 144.3 & 2.97 & $7.1 \%$ \\
\hline
\end{tabular}

where the conservative variables $\mathbf{Q}$, the fluxes $\mathbf{F}$ and $\mathbf{G}$ in the $x$ and $y$ directions, respectively, are

$$
\mathbf{Q}=(\rho, \rho u, \rho v, E)^{T}, \quad \mathbf{F}=\left(\rho u, \rho u^{2}+P, \rho u v,(E+P) u\right)^{T}, \quad \mathbf{G}=\left(\rho v, \rho u v, \rho v^{2}+P,(E+P) v\right)^{T},
$$

and the equation of state (EOS) is

$$
P=(\gamma-1)\left(E-\frac{1}{2} \rho\left(u^{2}+v^{2}\right)\right), \quad \gamma=1.4
$$

The $\rho, u, v, P$, and $E$ are the density, velocity in $x$ - and $y$-directions, pressure and total energy respectively.

\subsubsection{One-Dimensional Shock-Density Wave Interaction Problem}

This test case proposed in [21] describes the interaction of a right moving shock with an oscillatory smooth wave. Its initial condition is given by

$$
(\rho, u, p)=\left\{\begin{array}{ll}
\left(\frac{27}{7}, \frac{4 \sqrt{35}}{9}, \frac{31}{3}\right) & \text { if } x<-4 \\
(1+0.2 \sin (5 x), 0,1) & \text { if } x>-4
\end{array}, \quad x \in[-5,5] .\right.
$$

The boundary conditions are reflective boundary conditions. The solution is simulated on a mesh with $N=400$ and $N=800$ cells, until the time $t=1.8$. Fig. 11 shows the density $\rho$ and the temporal history of the WENO-Z reconstruction at each grid point. In the case of low mesh resolution $N=400$, high frequency waves can not be fully recognized. With the increase of mesh resolution $N=800$, high frequency waves can be accurately identified. The CPU timings and WENO percentage are shown in Table $\mathrm{V}$, the Hybrid-ANN scheme is as efficient as the Hybrid-MR scheme with $\epsilon_{M R}=5 \times 10^{-3}$, especially in the high mesh resolution case.

\begin{tabular}{|c|c|c|c|c|c|c|c|}
\hline \multirow{2}{*}{$\mathrm{N}$} & WENO & \multicolumn{3}{|c|}{ Hybrid-MR } & \multicolumn{3}{|c|}{ Hybrid-ANN } \\
\hline & seconds & seconds & SF & percentage & seconds & $\mathrm{SF}$ & percentage \\
\hline$N=400$ & 0.75 & 0.56 & 1.34 & $17.5 \%$ & 0.59 & 1.27 & $11.9 \%$ \\
\hline$N=800$ & 1.92 & 1.47 & 1.31 & $8.99 \%$ & 1.49 & 1.29 & $4.49 \%$ \\
\hline
\end{tabular}

Table V: One-dimensional shock-density wave interaction problem. The CPU timings in seconds and speedup factors of the WENO-Z and Hybrid schemes. 

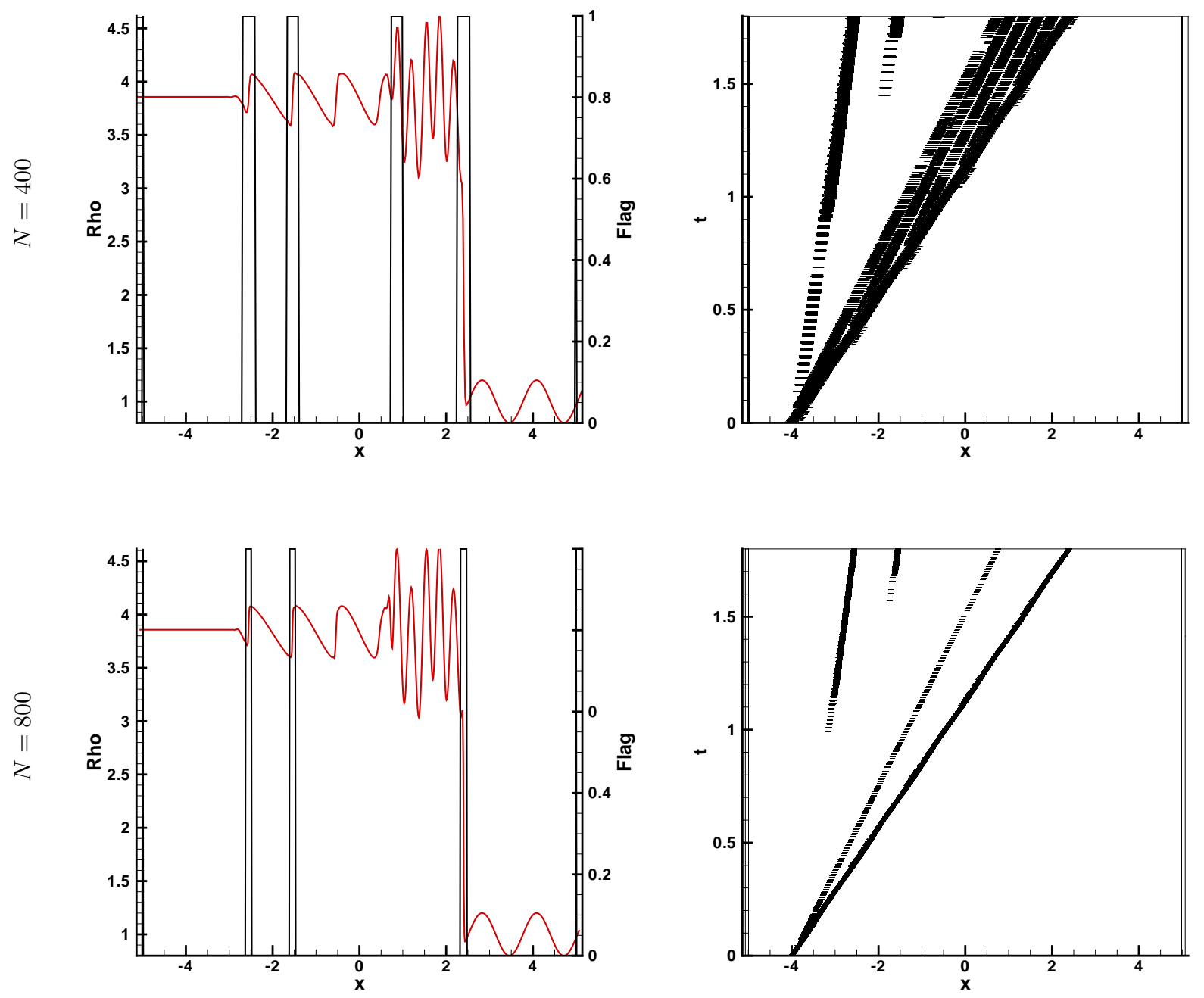

Figure 11: (Left) The density $\rho$ and WENO Flag of the shock-density wave interaction problem as computed by the Hybrid-ANN scheme at $t=1.8$, and (Right) temporal history of the WENO-Z reconstruction as computed by the Hybrid-ANN scheme, using (Top) $N=400$ and (Bottom) $N=800$. 


\subsubsection{Two-Dimensional Riemann Initial Value Problem}

To examine the performance of the Hybrid scheme for higher dimensional problems, we solve the classical Riemann initial value problem. According to [18], there are 19 genuinely different admissible configurations for polytropic gas, separated by the three types of one-dimensional centered waves. We refer readers to [18] for details. Here, we show the representative results of configurations 3 with a mesh resolution of $N \times M=400 \times 400$, the center is $\left(x_{0}, y_{0}\right)=(0.8,0.8)$ to allow a longer time simulation for a further development of the fine scale structures. The initial conditions are

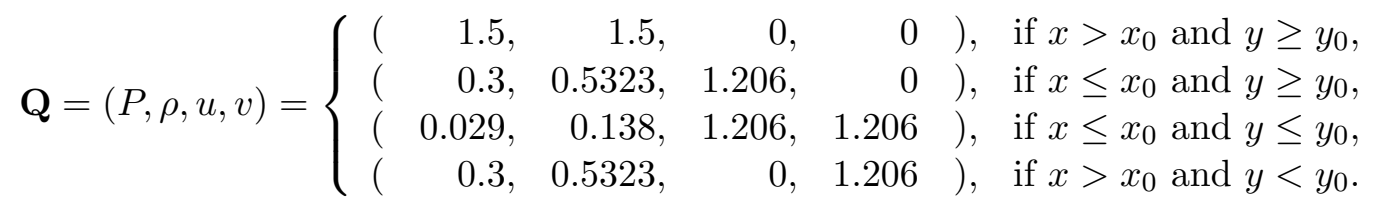

The density with flooded contours and lines are shown in Fig. 12. The large scale structures of the flow agree well with those in the literature. We remark that the WENO flag Flag in $x$ - and $y$-directions are very sharp with only a few grid points contained in each segment showing the accuracy of the ANN shock detection algorithm. Table VI presents the CPU timings along with the speedup factor in the simulation of configuration 3 with the WENO-Z, Hybrid-MR and Hybrid-ANN schemes. From the results, we can see that the Hybrid schemes are substantially faster than the WENO-Z scheme with a speedup factor increased about two as the mesh resolution increases, and the Hybrid-ANN scheme is faster with less WENO percentage.

Table VI: The CPU timings, percentage of the WENO-Z reconstruction, and speedup factors (SF) of the WENO-Z and Hybrid schemes.

\begin{tabular}{|c|c|c|c|c|c|c|c|}
\hline \multirow{2}{*}{$N \times M$} & \multirow{2}{*}{$\begin{array}{c}\text { WENO } \\
\text { seconds }\end{array}$} & \multicolumn{3}{|c|}{ Hybrid-MR } & \multicolumn{3}{|c|}{ Hybrid-ANN } \\
\hline & & seconds & SF & percentage & seconds & SF & percentage \\
\hline $200 \times 200$ & 218.5 & 151.3 & 1.44 & $33.3 \%$ & 112.6 & 1.94 & $11.1 \%$ \\
\hline $400 \times 400$ & 1863 & 888.2 & 2.09 & $19.2 \%$ & 717.7 & 2.59 & $5.81 \%$ \\
\hline
\end{tabular}

\subsubsection{Two-Dimensional Mach 10 Double Mach Reflection Problem}

To illustrate the efficiency of the Hybrid schemes in a more practical problem, we applied both Hybrid schemes to solve the two-dimensional Mach 10 double Mach reflection (DMR) problem. In this problem, a Mach 10 normal shock wave impinges onto a wedge with a given angle of inclination. By changing the frame of reference to the surface of the wedge, we setup the computational domain as $[0,4] \times[0,1]$. The Mach 10 oblique shock makes contact with the lower domain boundary at 60 degree angle with the horizontal $x$-axis. The initial conditions are

$$
Q=(\rho, u, v, P)=\left\{\begin{array}{cl}
(8,8.25 \cos \theta,-8.25 \sin \theta, 116.5), & x<x_{0}+y / \sqrt{3} \\
(1.4,0,0,1), & x \geq x_{0}+y / \sqrt{3}
\end{array}\right.
$$

with $x_{0}=\frac{1}{6}$ and $\theta=\pi / 6$. Supersonic inflow and free-stream outflow boundary conditions are specified at $x=0$ and $x=4$, respectively. At the lower boundary $y=0$, reflective boundary conditions are applied in 
Density $\rho$

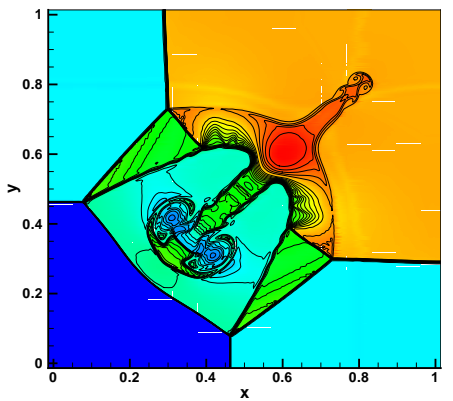

$\operatorname{Flag}_{x}$

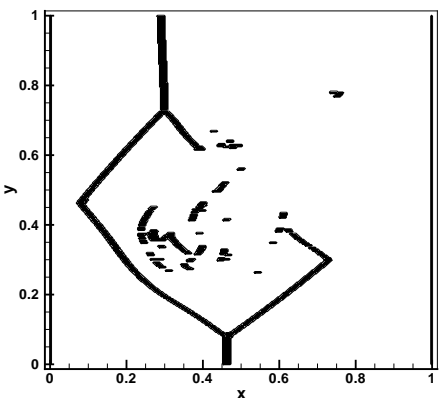

$\operatorname{Flag}_{y}$

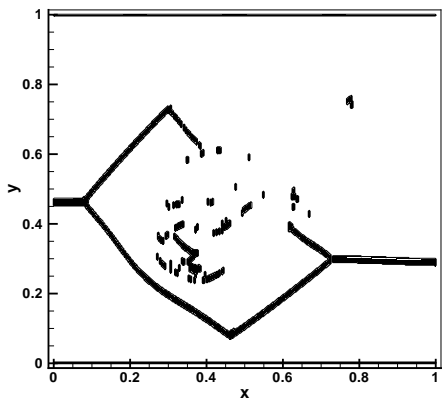

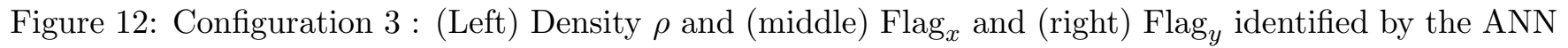
edge detector under resolution $400 \times 400$ at time $t=0.8$.

the interval $\left[x_{0}, 4\right]$. At the upper boundary $y=1$, the exact solution of the Mach 10 moving oblique shock is imposed. The problem and its numerical results are well documented in the literature [25].

We run both the Hybrid-MR and Hybrid-ANN schemes with mesh resolution $N \times M=400 \times 100$ uniform cells to the final time $t_{f}=0.2$. The density flooded contours and lines and the WENO Flag in $x$ - and $y$-directions of the Hybrid-ANN scheme with different mesh resolutions are shown in the Fig. 13. The CPU timings, percentage of the WENO-Z reconstruction, and speedup factors are shown in Table VII. Generally speaking, the Hybrid-ANN scheme captures the high gradients more accurate than the Hybrid-MR scheme.

Table VII: The CPU timings, percentage of the WENO-Z reconstruction, and speedup factors (SF) of the WENO-Z and Hybrid schemes, using mesh resolutions (Top) $N \times M=400 \times 100$ and (Bottom) $N \times M=800 \times 200$.

\begin{tabular}{|c|c|c|c|c|c|c|c|}
\hline \multirow{2}{*}{$N \times M$} & WENO & \multicolumn{3}{|c|}{ Hybrid-MR } & \multicolumn{3}{|c|}{ Hybrid-ANN } \\
\hline & seconds & seconds & $\mathrm{SF}$ & percentage & seconds & $\mathrm{SF}$ & percentage \\
\hline $400 \times 100$ & 150.8 & $\overline{92.6}$ & 1.63 & $26.5 \%$ & 75.3 & 2.00 & $11.8 \%$ \\
\hline $800 \times 200$ & 1242 & 527 & 2.56 & $14.3 \%$ & 465.8 & 2.67 & $6.1 \%$ \\
\hline
\end{tabular}

\section{Conclusion}

In this work, we develop a new edge detector without problem dependent parameter based on a simple back propagation artificial neural network (ANN), which only contains two hidden layers with $\{8,4\}$ neurons. The ANN edge detector has been successfully employed in the image edge detection and the high order hybrid Compact-WENO (Hybrid) scheme for the solutions of hyperbolic conservation laws respectively. It can capture the image edges accurately with fewer grid points than the multi-resolution (MR) analysis. Furthermore, the Hybrid scheme with the new edge detector use similar CPU time to that with the MR analysis in solving the Euler and shallow water equations with the solutions containing discontinuities. But the number of non-smooth grid points captured by the new edge detector is only half of that detected by the MR analysis with a user tunable parameter. 

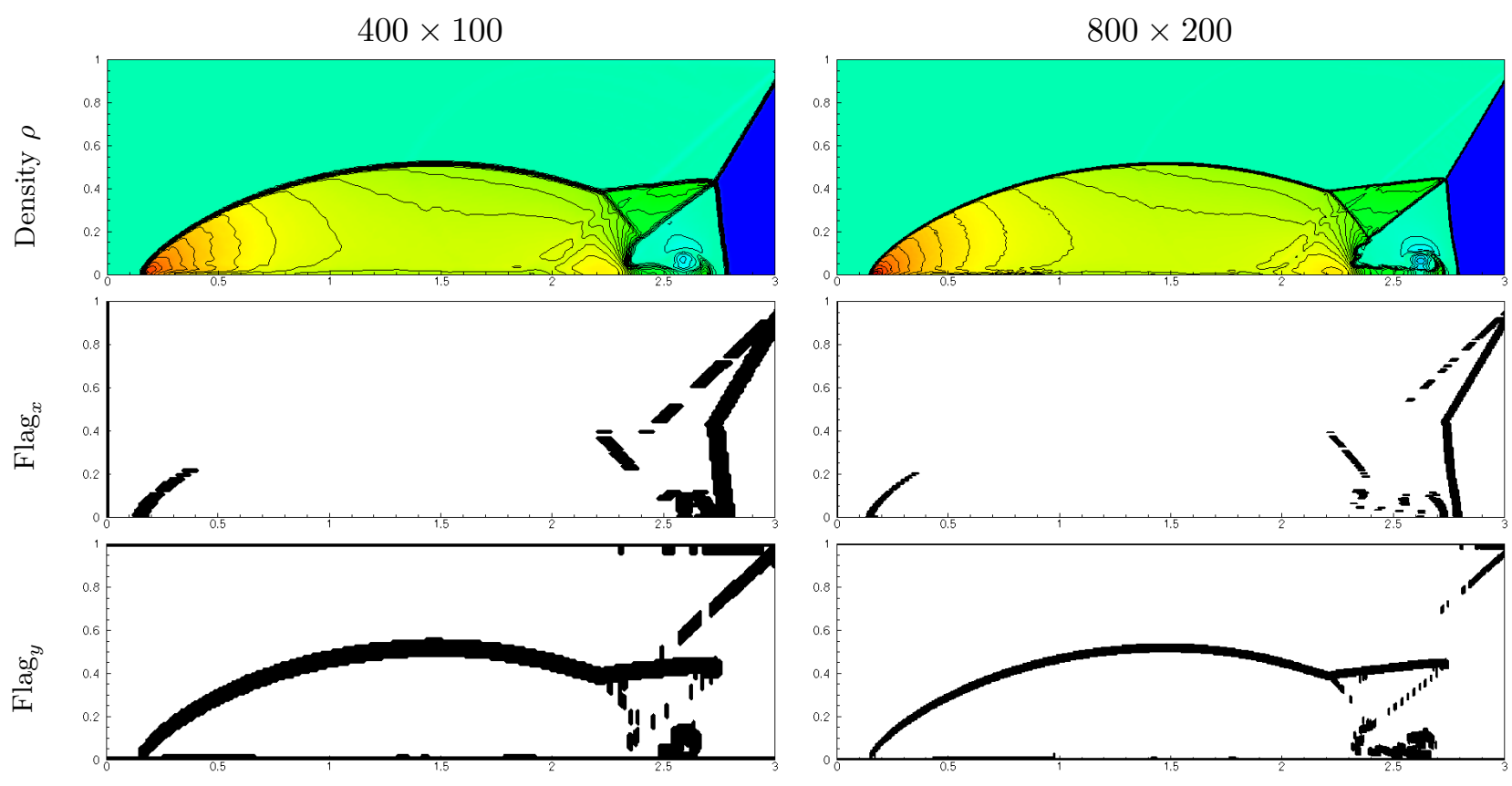

Figure 13: The Mach 10 double Mach shock reflection problem. (Top) Density $\rho$ and (middle) Flag ${ }_{x}$ and

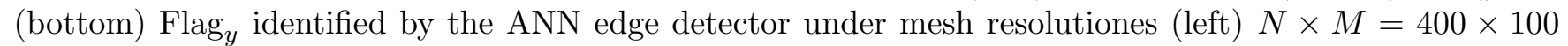
and (right) $N \times M=800 \times 200$ at time $t=0.2$.

\section{Acknowledgments}

The authors would like to acknowledge the funding support of this research by the National Natural Science Foundation of China (11871443) and Shandong Provincial Natural Science Foundation (ZR2017MA016). The author (Don) also likes to thank the Ocean University of China for providing the startup funding (201712011) that is used in supporting this work.

\section{Appendix}

\section{A Multi-resolution analysis}

The general idea of multi-resolution (MR) analysis [10] is to generate a coarser grid of averages of the point values of a function and measure the differences (MR coefficients) $d_{i}$ between the interpolated values from this sub-grid and the point values themselves. Given an initial number of the grid points $N_{0}+1$ and grid spacing $\Delta x_{0}$ in the domain $[0,1]$, we shall consider a set of nested dyadic grids up to $\operatorname{level} L<\log _{2} N_{0}$,

$$
G^{k}=\left\{x_{i}^{k}, \quad i=0, \ldots, N_{k}\right\}, \quad 0 \leq k \leq L,
$$


where $x_{i}^{k}=i \Delta x_{k}$ with $\Delta x_{k}=2^{k} \Delta x_{0}, N_{k}=2^{-k} N_{0}$ and the cell averages of function $u$ at $x_{i}^{k}$ :

$$
\bar{u}_{i}^{k}=\frac{1}{\Delta x_{k}} \int_{x_{i-1}^{k}}^{x_{i}^{k}} u(x) d x .
$$

Let $\tilde{u}_{2 i-1}^{k}$ be the approximation to $\bar{u}_{2 i-1}^{k}$ by a unique polynomial of degree $n_{M R}=2 s$ that interpolates $\bar{u}_{i+l}^{k},|l| \leq s$ at $x_{i+l}^{k}$, where $q=2 s+1$ is the order of approximation .

The approximation error (or multi-resolution coefficients), taking $k=1$ for a single-level MR, $d_{i}=\bar{u}_{2 i-1}^{0}-$ $\tilde{u}_{2 i-1}^{0}$ at $x_{i}$, has the property that if $u(x)$ is a $C^{p-1}$ function, then

$$
d_{i} \approx\left\{\begin{array}{cc}
{\left[u_{i}^{(p)}\right] \Delta x_{1}^{p}} & p \leq q \\
u_{i}^{(q)} \Delta x_{1}^{q} & p>q
\end{array},\right.
$$

where $[\cdot]$ and $(\cdot)$ denote the jump $\left(\left[f_{i}\right]=\left|f_{i+1}-f_{i}\right|\right)$ and the derivatives of the function $\left(f_{i}^{(p)}=\frac{d^{p}}{d x^{p}} f\left(x_{i}\right)\right)$, respectively. The MR coefficient $d_{i}$ measures how close the data at the finer grid can be interpolated by the data at the coarser grid.

Hence, one can determine the local smoothness of the function at a given point using the MR coefficients $d_{i}$ and set the MR flag, Flag $_{i}$ at $x_{i}$, as

$$
\text { Flag }_{i}=\left\{\begin{array}{lll}
1, & \left|d_{i}\right|>\epsilon_{M R} & \text { Discontinuous } \\
0, & \text { otherwise } & \text { Smooth }
\end{array}\right.
$$

where $\epsilon_{M R}$ is the MR tolerance and it is a user tunable parameter. $\epsilon_{M R}=10^{-3}$ is used in this study without state otherwise.

\section{B Compact Finite Difference Schemes}

The sixth order compact finite difference scheme [13] can be written compactly as

$$
\mathbf{A g}^{\prime}=\mathbf{B g}+\mathbf{b}
$$

where $\mathbf{A}$ and $\mathbf{B}$ are the banded coefficient matrices,

$$
\mathbf{A}=\left(\begin{array}{cccc}
1 & 1 / 3 & & \\
1 / 3 & 1 & 1 / 3 & \\
& \ddots & \ddots & \\
& 1 / 3 & 1 / 3 & 1 / 3 \\
& & 1 / 3 & 1
\end{array}\right), \quad \mathbf{B}=\frac{1}{36 \Delta x}\left(\begin{array}{cccccc}
0 & 28 & 1 & & & \\
-28 & 0 & 28 & 1 & & \\
-1 & -28 & 0 & 28 & 1 & \\
& \ddots & \ddots & \ddots & \ddots & \ddots \\
& & -1 & -28 & 0 & 28 \\
& & & -1 & -28 & 0
\end{array}\right)
$$

The vector $\mathbf{b}$ is

$$
\mathbf{b}=\frac{1}{36 \Delta x}\left(-g_{-1}-28 g_{0},-g_{0}, 0, \cdots, 0, g_{N}, 28 g_{N}+g_{N+1}\right)^{\mathrm{T}}-\frac{1}{3}\left(g_{0}^{\prime}, 0,0, \cdots, 0,0, g_{N}^{\prime}\right)^{\mathrm{T}}
$$

where $g_{-1}=g\left(x_{0}-\Delta x\right)$ and $g_{N+1}=g\left(x_{N}+\Delta x\right)$ are the ghost points. 


\section{Weighted Essentially Non-Oscillatory Schemes}

We briefly review the fifth order WENO-Z nonlinear scheme [6, 2]. The 5-point global stencil $S^{5}=$ $\left(x_{i-2}, \cdots, x_{i+2}\right)$ is subdivided into three 3 -point substencils $S_{k}=\left(x_{i+k-2}, x_{i+k-1}, x_{i+k}\right),(k=0,1,2)$. The fifth degree polynomial approximation $\mathbb{P}_{i+\frac{1}{2}}^{5}$ in the stencil $S^{5}$ is built through the convex combination of three second order interpolation polynomials $\mathbb{P}^{2}(x)$ in each substencil $S_{k}$ at the cell boundaries $x_{i+\frac{1}{2}}$.

$$
\mathbb{P}_{i+\frac{1}{2}}^{5}=\sum_{k=0}^{2} \omega_{k} \mathbb{P}^{2}\left(x_{i+\frac{1}{2}}\right)
$$

where

$$
\alpha_{k}=d_{k}\left(1+\left(\frac{\tau_{5}}{\beta_{k}+\varepsilon}\right)^{p}\right), \quad \omega_{k}=\frac{\alpha_{k}}{\sum_{j=0}^{r-1} \alpha_{j}} .
$$

Here $\tau_{5}=\left|\beta_{0}-\beta_{2}\right|$. The sensitivity and power parameters are $\epsilon=10^{-12}$ and $p=2$ respectively. The ideal weights $d_{k}$ are $d_{0}=\frac{1}{10}, d_{1}=\frac{3}{5}, d_{2}=\frac{3}{10}$. The regularity (smoothness) in the substencil is measured by the (lower order local) smoothness indicators $\beta_{k}$, which are given by

$$
\beta_{k}=\sum_{l=1}^{r-1} \Delta x^{2 l-1} \int_{x_{i-\frac{1}{2}}}^{x_{i+\frac{1}{2}}}\left(\frac{d^{l}}{d x^{l}} \hat{f}^{k}(x)\right)^{2} d x .
$$

\section{References}

[1] K. Anastasiou, C. T. Chan, Solution of the 2D shallow water equations using the finite volume method on unstructied triangular meshes. Int. J. Numer. Methods Fluid 24, (1997) 1225-1245.

[2] R. Borges, M. Carmona, B. Costa and W.S. Don, An improved weighted essentially non-oscillatory scheme for hyperbolic conservation laws, J. Comput. Phys. 227 (2008) 3191-3211.

[3] M. Castro, B. Costa and W. S. Don, High Order Weighted Essentially Non-Oscillatory WENO-Z Schemes for Hyperbolic Conservation Laws, J. Comput. Phys. 230 (2011) 1766-1792.

[4] B. Costa and W.S. Don, High order Hybrid Central-WENO finite difference scheme for conservation laws, J. Comput. Appl. Math. 204 No. 2 (2007) 209-218.

[5] B. Costa and W.S. Don, Multi-domain hybrid spectral-WENO methods for hyperbolic conservation laws, J. Comput. Phys. 224 (2007) 970-991.

[6] W. S. Don and R. Borges, Accuracy of the Weighted Essentially Non-Oscillatory Conservative Finite Difference Schemes, J. Comput. Math. 205 (2013) 347-372.

[7] W. S. Don, Z. Gao, P. Li, X. Wen, Hybrid compact-WENO finite difference scheme with conjugate Fourier shock detection algorithm for hyperbolic conservation laws, SIAM J. Sci. Comput. 38 (2016) A691-A711. 
[8] Z. Gao and W. S. Don, Mapped Hybrid Central-WENO Finite Difference Scheme for Detonation Waves Simulations, J. Sci. Comput. 55 (2012) 351-371.

[9] Z. Gao, X. Wen, W. S. Don, Enhanced Robustness of the Hybrid Compact-WENO Finite Difference Scheme for Hyperbolic Conservation Laws with Multi-resolution Analysis and Tukeys Boxplot Method, J. Sci. Comput. 73 (2017) 736-C752.

[10] A. Harten, High resolution schemes for hyperbolic conservation laws, J. Comput. Phys. 49 (1983) $357-393$.

[11] G. S. Jiang and C. W. Shu, Efficient Implementation of Weighted ENO Schemes, J. Comput. Phys. 126 (1996), 202-228.

[12] T. Kohonen, An introduction to neural computing, Neural Networks, 1(1) (1988) 3-16.

[13] S.A. Lele, Compact finite difference schemes with spectral-like resolution, J. Comput. Phys. 103 No.1 (1992) 16-42.

[14] G. Li, C. Lu and J. Qiu, Hybrid Well-balanced WENO Schemes with Different Indicators for Shallow Water Equations, J. Sci. Comput. 51 (2012), 527-559.

[15] Y. Niu, Z. Gao, W.S. Don, S.S. Xie and P. Li, Hybrid Compact-WENO Finite Difference Scheme For Detonation Waves Simulations, Spectral and High Order Methods for Partial Differential Equations ICOSAHOM 2014. R. M. Kirby, M. Berzins, J. S. Hesthaven (eds.), Lect. Notes Comput. Sci. Eng. 106 (2015), Springer.

[16] S. Pirozzoli, Conservative Hybrid Compact-WENO Schemes for Shock-Turbulence Interaction, J. Comput. Phys. 178 No. 1 (2002), 81-117.

[17] D. Ray, J. S.Hesthaven, An artificial neural network as a troubled-cell indicator J. Comput. Phys. 367 (2018), 166-191.

[18] C. W. Schulz-Rinne, Classification of the Riemann problem for Two-dimensional Gas Dynamics, SIAM J. Math. Anal. 24 (1993) 76-88.

[19] D. E. Rumelhart, J. L. Mcclelland, On learning the past tenses of English verbs // Parallel distributed processing: explorations in the microstructure of cognition, vol. 2. MIT Press, 1986.

[20] K. Shahbazi, J. Hesthaven, X. Zhu, Multi-dimensional hybrid Fourier continuation-WENO solvers for conservation laws, J. Comput. Phys. 253 (2013) 209C-225.

[21] C.-W. Shu, S. Osher, Efficient implementation of essentially non-oscillatory shock-capturing schemes, II, J. Comput. Phys. 83 (1989) 32-78.

[22] C.W. Shu, High order Weighted Essentially Nonoscillatory Schemes for convection dominated problems, SIAM Rev. 51 No. 1 (2009) 82-126.

[23] O. Vasilyev, T. Lund and P. Moin, A General Class of Commutative Filters for LES in Complex Geometries, J. Comput. Phys.146 No. 1 (1998) 82-104. 
[24] S. Vukovic and L. Sopta, ENO and WENO schemes with the exact conservation property for onedimensional shallow water equations, SJ. Comput. Phys. 179 (2002) 593-621.

[25] P. Woodward and P. Collela, The numerical simulation of two dimensional fluid flow with strong shocks, J. Comput. Phys. 54 (1984) 115-173.

[26] Y. L. Xing, C.-W. Shu, High order finite difference WENO schemes with the exact conservation property for the shallow water equations, J. Comput. Phys. 208 (2005) 206-227.

[27] Q.Q. Zhu, Z. Gao, W.S. Don, X.Q. Lv, Well-balanced hybrid compact-WENO schemes for shallow water equations, Appl. Numer. Math. 112 (2017) 65-78. 